\title{
The Correlation Between Low Serum T3 Levels and All-Cause and Cardiovascular Mortality in Peritoneal Dialysis Patients
}

\author{
Ling-Cang $\mathrm{Xu} \mathbb{D}^{1,2}$ \\ Fang-Fang Zhou ${ }^{1,2}$ \\ Meng $\mathrm{Li}^{1,2}$ \\ Zhi-Wei Dai ${ }^{1,2}$ \\ Ke-Dan Cai ${ }^{1,2}$ \\ Bei-Xia Zhu',2 \\ Qun Luo ${ }^{1,2}$
}

'Department of Nephrology, Hwa Mei Hospital, University of Chinese Academy of Sciences, Ningbo, Zhejiang Province, 3I50 I0, People's Republic of China; ${ }^{2}$ Ningbo Institute of Life and Health Industry, University of Chinese Academy of Sciences, Ningbo, Zhejiang Province, 315010, People's Republic of China
Correspondence: Qun Luo Department of Nephrology, Hwa Mei Hospital, University of Chinese Academy of Sciences, No. 4I, Northwest Street, Ningbo, Zhejiang Province, 315010, People's Republic of China $\mathrm{Tel} / \mathrm{Fax}+86-574-83870217$

Email nbeyluoqun@126.com; luoqun@ucas.ac.cn
Objective: This study is to investigate the correlation between serum triiodothyronine (T3) levels and all-cause and cardiovascular mortality in PD patients.

Methods: A total of 376 end-stage renal disease (ESRD) patients who started maintenance PD treatment in the Department of Nephrology in our hospital and stable treatment for $\geq 3$ months were selected, and the total T3 (TT3) and free T3 (FT3) levels were determined. Among them, 168 cases with FT3 $<3.5 \mathrm{pmol} / \mathrm{L}$ and/or TT3 $<0.92 \mathrm{nmol} / \mathrm{L}$ were divided into the low serum T3 level group, and the remaining 208 cases were divided into normal serum T3 level group. The Cox survival analysis method was used to analyze the correlation between low serum T3 levels and all-cause and cardiovascular mortality in PD patient.

Results: Compared with the normal serum T3 level group, patients with low serum T3 levels had higher systolic blood pressure and a higher proportion of heart disease, and lower levels of total T4, free T4, hemoglobin, serum albumin, blood calcium, serum total bilirubin, alanine aminotransferase, and 24-h urine volume (all $\mathrm{P}<0.05$ ). Binary Logistic regression analysis showed that heart disease ( $\mathrm{P}=0.003$, OR: 2.628, 95\% CI: 1.382-4.997) and high TT4 level ( $<<0.001$, OR: 0.968, 95\% CI: 0.956-0.979) were related to low serum T3 levels in PD patients. Multivariate Cox regression analysis showed that low serum FT3 level was an independent risk factor for all-cause death in PD patients $(\mathrm{HR}=0.633,95 \% \mathrm{CI}=0.431-$ 0.930; $\mathrm{P}<0.020)$.

Conclusion: Low serum T3 levels in PD patients were associated with heart disease and TT4 levels. Low serum FT3 levels were associated with the risk of all-cause death in PD patients.

Keywords: serum T3 level, peritoneal dialysis, all-cause death, cardiovascular death, correlation

\section{Introduction}

The thyroid is the biggest endocrine organ of the human body. The thyroid hormones it secretes play an essential role in kidney development, maintaining water and electrolyte balance, and kidney function. ${ }^{1}$ Under conditions such as renal hypofunction, it will also have a greater effect on the synthesis, secretion and clearance of thyroid hormones. ${ }^{2}$ On the other hand, thyroid hormones significantly impact renal development and kidney dynamics., ${ }^{3,4}$ Elevated TSH, FT4, and reduced T3 levels were associated with reduced kidney function. ${ }^{5}$ In the previous study, serum FT3 could be a potential biomarker for chronic kidney disease (CKD) among Chinese adults. ${ }^{6}$ 
End-stage renal disease (ESRD) occurs while CKD reaches an advanced state. Peritoneal dialysis (PD) is an effective renal replacement therapy for patients with ESRD. Studies ${ }^{7,8}$ have shown that abnormal thyroid function is closely related to the poor prognosis of PD patients. However, the correlation between total triiodothyronine (TT3), free triiodothyronine (FT3) or other laboratory indicators and all-cause death and cardiovascular disease (CVD) death in PD patients has not yet reached consensus in the current research. Some studies ${ }^{7,9}$ pointed out that the incidence of low T3 syndrome in PD patients was relatively high, which was closely related to all-cause and CVD deaths and represented a factor directly related to cardiac complications in PD patients. Tian et al ${ }^{10}$ pointed out that FT3 is the only thyroid hormone that can independently predict all-cause death in maintenance peritoneal dialysis patients. The mechanism may be related to the malnutrition-inflammation syndrome, which has been proven to be an extremely unfavorable factor affecting the prognosis of ESRD patients. ${ }^{11}$

In this study, the clinical data of 376 ESRD patients receiving PD treatment in our hospital were retrospectively analyzed, and the correlation between low T3 levels and the risk of all-cause and CVD deaths in PD patients was explored.

\section{Materials and Methods}

\section{Study Design and Patient Population}

The subjects of the study were patients who had an indwelling peritoneal dialysis tube in the Department of Nephrology, Hwa Mei Hospital, University of Chinese Academy of Sciences from October 1, 2006 to September 30, 2020 for PD treatment. The inclusion criteria were: age $\geq 18$ years; PD treatment $\geq 3$ months. The exclusion criteria were: out-of-hospital catheterization, previous history of kidney transplantation; previous hemodialysis $\geq 3$ months; CVD occurrence within 3 months; patients with rheumatic or organic heart disease; patients with chronic liver disease and severe liver dysfunction; those who have had blood transfusion within 3 months before the study; those with incomplete clinical data; those who have had a history of thyroid disease or hypothalamopituitary disorders now or in the past; determined thyroid hormone levels (within 1 month), and took medicines that may affect the secretion and metabolism of thyroid hormone. This study was approved by the ethics committee of Hwa Mei Hospital, University of Chinese Academy of
Sciences (No.PJ-KY-NBEY-2016-016-01) and complied with the principles of the Declaration of Helsinki for medical research. Patient consent was waived because of the retrospective nature of the study and the analysis used anonymous clinical data.

\section{Data Collection}

All patients were followed up to death, transferred to hemodialysis treatment, transferred to kidney transplantation, transferred to other dialysis centers, lost to follow-up or followed up until September 30, 2020 through telephone, outpatient, WeChat and other ways. We collected baseline data of all selected patients at the third month afterPD, including demographic data: gender, age, smoking, drinking, systolic blood pressure, diastolic blood pressure, heart rate, weight, height, body mass index (BMI), hypertension, diabetes, heart disease such as chronic heart failure, coronary artery disease, arrhythmia, heart valve and myocardial disease etc., cerebrovascular disease, and laboratory biochemical indicators: TT3, FT3, thyroidstimulating hormone (TSH), total thyroxine (TT4), free thyroxine (fT4), hemoglobin, serum albumin, alkaline phosphatase, blood creatinine, urea nitrogen, uric acid, blood calcium, blood phosphorus, C-reactive protein, serum total bilirubin, aspartate aminotransferase, alanine aminotransferase, parathyroid hormone value, low-density lipoprotein $\mathrm{C}$, triglycerides, total cholesterol, 24-h urine volume, glomerular filtration rate (eGFR), eGFR calculation referred to CKD-EPI formula. The thyroid hormone levels of included patients were determined by acridinium chemiluminescence with ADVIA centaur XP (Siemens Healthineers) in the same laboratory. The clinical outcome and cause of death of patients were recorded. The causes of death was jointly determined by the physician and the nurse. CVD death is caused by a range of diseases related to the circulatory system, containing coronary heart disease, cerebrovascular disease, and other conditions. ${ }^{12,13}$ The normal range of FT3 and TT3 are $3.5 \sim 6.5 \mathrm{pmol} / \mathrm{L}$, and $0.92 \sim 2.79 \mathrm{nmol} / \mathrm{L}$ respectively in our laboratory, which is same with other laboratory in China. ${ }^{9}$ In this study, patients with serum $\mathrm{FT} 3<3.5 \mathrm{pmol} / \mathrm{L}$ and/or TT $3<0.92 \mathrm{nmol} / \mathrm{L}$ were divided into low serum T3 level group.

\section{Statistical Analysis}

SPSS 25.0 software was used for statistical analysis of the data. The measurement data conformed to the normal distribution were expressed as mean \pm standard 
deviation $\left(x^{-} \pm s\right)$, and $t$-test was used for comparison between two groups. The measurement data that did not conform to the normal distribution were expressed as $M\left(P_{25}-P_{75}\right)$, and Mann-Whitney $U$-test was used for comparison between two groups. The count data was expressed as rate (\%), and the two groups were compared using $\chi^{2}$ test. The variables with significantly difference in unavailable Cox regression analysis and gender, BMI, and cerebrovascular disease were included to perform multivariable model. Binary logistic regression analysis method was used to analyze the influencing factors of low T3 level, and the statistical power of influencing factors were determined by $\mathrm{G}$ Power 3.1 . The Kaplan-Meier survival analysis method was used to compare the survival and prognosis of patients, and Cox survival analysis method was used to analyze the correlation between low serum T3 levels and all-cause and CVD death in PD patients. $P<0.05$ indicates that the difference is statistically significant.

\section{Results}

\section{Baseline Demographic and Clinical Characteristics of Patients}

A total of 376 patients were included in this study. Average age of patients was $(57.80 \pm 15.13)$ years, included 217 (57.71\%) males and 116 (30.85\%) diabetic patients. Compared with the normal serum T3 level group, patients with low serum T3 levels had higher systolic blood pressure and a higher proportion of heart disease, and lower levels of TT4, fT4, hemoglobin, serum albumin, blood calcium, serum total bilirubin, alanine aminotransferase, and 24-h urine volume (all $P<0.05$ ). There was no significant difference in the other baseline data (all $P>0.05$ ) (Table 1).

\section{Binary Logistic Regression Analysis of Factors Related to Low Serum T3 Levels}

The results showed that systolic blood pressure $(\mathrm{P}=0.049$, OR: 1.009 , 95\% CI: $1.000-1.018)$, heart disease $(P=0.003$, OR: 2.628, 95\% CI: 1.382-4.997) and high TT4 level $(P<0.001$, OR: 0.968 , 95\% CI: 0.956-0.979) were related to low serum T3 levels in PD patients (Table 2). The statistical power of heart disease and TT4 level were 0.9999427 and 0.9999480 , respectively. However, the statistical power of systolic blood pressure was low as 0.6375900 .

\section{The Correlation Between Low Serum T3 Levels and All-Cause and CVD Deaths in PD Patients}

The end of follow-up for this study was as of September 30, 2020. There were 70 (18.62\%) deaths, 12 (3.19\%) cases of kidney transplants, $40(10.64 \%)$ cases of HD transfer, and $5(1.33 \%)$ cases lost to follow-up. Fiftyone $(13.56 \%)$ cases were transferred to other dialysis centers, and the remaining 198 (52.52\%) cases continued to be followed up. Among the 70 deaths, 37 (52.56\%) cases died of CVD, which containing 19 (27.14\%) cases with coronary heart disease, $9(12.86 \%)$ cases with cerebrovascular disease, $6(8.57 \%)$ cases with heart failure and 3 (4.29\%) cases with sever arrhythmia. Beside of the CVD death, $10(14.29 \%)$ cases died of infection, $6(8.57 \%)$ cases died of malignant tumors, $1(1.43 \%)$ case died of gastrointestinal bleeding, 5 (7.14\%) cases died of malnutrition, and 11 (15.71\%) cases died of other causes.

The Kaplan-Meier curves of patients with different T3 levels showed that the 1,3, and 5-year cumulative allcause mortality after the onset of PD in the serum T3 level group were $7.74 \%$ (13/168), 19.05\% (32/168) and 23.21 (39/168), respectively; for the normal serum T3 level group, the cumulative all-cause mortality rates were $2.88 \%(6 / 208), 4.81 \%(10 / 208), 6.73 \%$ (14/208), and the survival rate of the low serum T3 level group was significantly lower than the normal serum T3 level group (Log rank test $\chi^{2}=29.697, P<0.001$ ) (Figure 1). The 1, 3, and 5-year cumulative CVD mortality in the low serum T3 level group were $4.76 \%(8 / 168), 10.12 \%(17 / 168)$, and $13.10 \%(22 / 168)$, respectively. For the normal serum T3 level group, the cumulative CVD mortality were 1.92\% (4/ 208), $2.40 \%(5 / 208)$, and $2.88 \%(6 / 208)$. The survival rate of the low serum T3 level group was the normal serum T3 level group (Log rank test $\left.\chi^{2}=19.454, \quad P<0.001\right)$ (Figure 2).

\section{The Predictive Value of Serum T3 Level on the Prognosis of PD Patients}

After adjusting for patient demographics and important laboratory indicators, the multivariate Cox risk regression model (Tables 3 and 4) showed that low serum FT3 level was an independent risk factor for all-cause death in PD patients $(\mathrm{HR}=0.633,95 \% \mathrm{CI}=0.431-0.930 ; P=0.020)$. However, serum FT3 was not a significant independent risk factor for CVD death in PD patients $(\mathrm{HR}=1.054$, $95 \% \mathrm{CI}=0.608-1.826 ; P>0.05)$. 
Table I Comparison of Baseline Data Between Groups

\begin{tabular}{|c|c|c|c|c|c|}
\hline Indexes & $\mathbf{n}$ & $\begin{array}{l}\text { Normal Serum T3 Level Group } \\
\qquad(n=208)\end{array}$ & $\begin{array}{l}\text { Low Serum T3 Level Group } \\
\qquad(n=168)\end{array}$ & $t / \chi^{2}$ & $P$-value \\
\hline Gender [male, n (\%)] & 217 & II 6 (55.77) & $101(60.12)$ & 0.721 & 0.369 \\
\hline Age (years old) & & $56.61 \pm 14.82$ & $59.27 \pm 15.42$ & 1.704 & 0.089 \\
\hline Smoking [n (\%)] & 79 & $44(21.15)$ & $35(20.83)$ & 0.006 & 0.940 \\
\hline Drinking [n (\%)] & 46 & $24($ (I .54$)$ & $22(13.10)$ & 0.210 & 0.647 \\
\hline Systolic blood pressure $(\mathrm{mmHg})$ & & $145.77 \pm 24.58$ & $151.68 \pm 28.87$ & 2.144 & 0.033 \\
\hline Diastolic blood pressure $(\mathrm{mmHg})$ & & $81.75 \pm 17.77$ & $82.29 \pm 16.32$ & 0.307 & 0.759 \\
\hline Heart rate (times) & & $79.0 \pm 11.79$ & $78.96 \pm 10.49$ & 0.061 & 0.952 \\
\hline Weight (kg) & & $60.28 \pm 11.95$ & $61.47 \pm 12.35$ & 0.941 & 0.347 \\
\hline Height (m) & & $1.64 \pm 0.08$ & $1.65 \pm 0.07$ & 0.761 & 0.447 \\
\hline BMI $\left(\mathrm{kg} / \mathrm{m}^{2}\right)$ & & $22.33 \pm 3.76$ & $22.57 \pm 3.70$ & 0.615 & 0.539 \\
\hline Hypertension [n (\%)] & 332 & $184(88.46)$ & $148(88.10)$ & 0.025 & 0.988 \\
\hline Diabetes [n (\%)] & 116 & $62(29.81)$ & $54(32.14)$ & 2.590 & 0.274 \\
\hline Heart disease [n (\%)] & 74 & $28(\mid 3.46)$ & $46(27.38)$ & 11.391 & $<0.001$ \\
\hline Cerebrovascular disease $[\mathrm{n}(\%)]$ & 40 & $19(9.13)$ & $21(12.50)$ & 1.107 & 0.293 \\
\hline TSH (mlU/L) & & $2.74 \pm 0.73$ & $2.59 \pm 2.42$ & 1.014 & 0.311 \\
\hline TT3 (nmol/L) & & $1.38 \pm 0.28$ & $0.92 \pm 0.28$ & $|5.54|$ & $<0.001$ \\
\hline FT3 (pmol/L) & & $4.20 \pm 0.54$ & $3.02 \pm 0.56$ & 20.489 & $<0.001$ \\
\hline TT4 (nmol/L) & & $|04.69 \pm 27.4|$ & $88.95 \pm 27.10$ & 6.696 & $<0.001$ \\
\hline FT4 (pmol/L) & & $15.02 \pm 0.79$ & $14.30 \pm 3.29$ & 2.576 & 0.010 \\
\hline Hemoglobin $(g / L)$ & & $106.93 \pm 19.43$ & $102.45 \pm 22.12$ & 2.090 & 0.037 \\
\hline Serum albumin $(g / L)$ & & $34.13 \pm 5.68$ & $30.56 \pm 6.25$ & 5.786 & $<0.001$ \\
\hline Alkaline phosphatase (IU/L) & & $75.00(58.00,96.00)$ & $75.00(59.75,99.50)$ & - & $0.74 I$ \\
\hline Serum creatinine $(\mu \mathrm{mol} / \mathrm{L})$ & & $676.71 \pm 241.62$ & $632.95 \pm 231.00$ & 1.780 & 0.076 \\
\hline Urea nitrogen (mmol/L) & & $18.68 \pm 5.66$ & $17.78 \pm 6.23$ & $\mathrm{I} .474$ & 0.141 \\
\hline Uric acid $(\mu \mathrm{mol} / \mathrm{L})$ & & $394.12 \pm 95.99$ & $392.72 \pm 109.80$ & 0.132 & 0.895 \\
\hline Blood calcium (mmol/L) & & $2.13 \pm 0.22$ & $2.04 \pm 0.22$ & 3.754 & 0.000 \\
\hline Blood phosphorus (mmol/L) & & $1.40 \pm 0.36$ & $1.34 \pm 0.44$ & 1.473 & 0.142 \\
\hline$C$ reactive protein $(\mathrm{mg} / \mathrm{L})$ & & $8.7 \pm 16.74$ & $11.12 \pm 22.44$ & 1.200 & 0.231 \\
\hline Serum bilirubin $(\mu \mathrm{mol} / \mathrm{L})$ & & $6.25 \pm 4.84$ & $5.05 \pm 2.21$ & 2.979 & 0.003 \\
\hline Aspartate aminotransferase (IU/L) & & $20.35 \pm 9.72$ & $19.25 \pm 11.23$ & 1.014 & 0.311 \\
\hline Alanine aminotransferase (IU/L) & & $19.72 \pm 17.50$ & $16.01 \pm 10.26$ & 2.434 & 0.015 \\
\hline Parathyroid hormone value $(\mathrm{pg} / \mathrm{mL})$ & & $257.75 \pm 183.86$ & $262.47 \pm 217.64$ & 0.228 & 0.820 \\
\hline Low density lipoprotein $C(\mathrm{mmol} / \mathrm{L})$ & & $2.4 I \pm 0.88$ & $2.47 \pm 0.94$ & 0.617 & 0.538 \\
\hline Triglyceride (mmol/L) & & $1.75 \pm 1.66$ & $1.67 \pm 1.37$ & 0.540 & 0.590 \\
\hline Total cholesterol $(\mathrm{mmol} / \mathrm{L})$ & & $4.32 \pm 1.16$ & $4.43 \pm 1.24$ & 0.861 & 0.390 \\
\hline 24-h urine volume $(\mathrm{mL})$ & & $1097.54 \pm 459.28$ & $977.96 \pm 485.36$ & 2.324 & 0.021 \\
\hline eGFR $\left(\mathrm{mL} / \mathrm{min}\right.$ per $\left.1.73 \mathrm{~m}^{2}\right)$ & & $7.56 \pm 4.82$ & $8.11 \pm 4.29$ & 1.150 & 0.251 \\
\hline
\end{tabular}

Notes: TSH for Thyroid-stimulating hormone; TT3 for total triiodothyronine; FT3 for free triiodothyronine; TT4 for total thyroxine; FT4 for free thyroxine; eGFR for estimated Glomerular Filtration Rate.

\section{Discussion}

Previous studies have confirmed that PD patients usually have varying degrees of thyroid dysfunction, such as low T3 syndrome, subclinical hypothyroidism and clinical hypothyroidism, which is a protective factor for reduced body protein and energy expenditure. ${ }^{3,14,15}$ In recent years, as clinicians pay more and more attention to the management of thyroid function in PD patients, the risk awareness that abnormal thyroid function affecting the prognosis of
PD widely known. However, serum T3 level as one of the main reference indicators of thyroid function still lacks attention, and there is no consensus on serum T3 level for the clinical prognosis of PD patients.

There are many factors that affect the serum T3 level of PD patients. ${ }^{8,16,17}$ This study retrospectively analyzed the clinical data of $376 \mathrm{PD}$ patients and found that low serum T3 level was related to heart disease and TT4 level, which was similar to previous studies. ${ }^{18,19} \mathrm{~T} 3$ and $\mathrm{T} 4$ are 
Table 2 Binary Logistic Regression Analysis Results of Factors Related to Low Serum T3 Levels

\begin{tabular}{|c|c|c|c|c|c|c|}
\hline \multirow[t]{2}{*}{ Indexes } & \multicolumn{3}{|c|}{ Univariate Analysis } & \multicolumn{3}{|c|}{ Multivariate Analysis } \\
\hline & $\mathbf{P}$ & OR & $95 \% \mathrm{Cl}$ & $\boldsymbol{P}$ & OR & $95 \% \mathrm{Cl}$ \\
\hline Gender [male, n (\%)] & 0.396 & 0.836 & $0.554 \sim 1.264$ & 0.106 & 0.464 & $0.183 \sim 1.176$ \\
\hline Age (years old) & 0.090 & 1.012 & $0.998 \sim 1.026$ & 0.064 & 0.970 & $0.939 \sim 1.002$ \\
\hline Smoking [n (\%)] & 0.940 & 1.020 & $0.619 \sim 1.680$ & - & - & - \\
\hline Drinking [n (\%)] & 0.647 & 0.866 & $0.467 \sim 1.606$ & - & - & - \\
\hline Systolic blood pressure $(\mathrm{mmHg})$ & 0.034 & 1.008 & $1.001 \sim 1.016$ & 0.049 & 1.009 & $1.000 \sim 1.018$ \\
\hline Diastolic blood pressure $(\mathrm{mmHg})$ & 0.758 & 1.002 & $0.990 \sim 1.014$ & - & - & - \\
\hline Heart rate (times) & 0.952 & 0.999 & $0.981 \sim 1018$ & 0.401 & 1.018 & $0.977 \sim 1.060$ \\
\hline Weight (kg) & 0.347 & 1.008 & $0.991 \sim 1.025$ & - & - & - \\
\hline Height (m) & 0.446 & 1.010 & $0.984 \sim 1.038$ & - & - & - \\
\hline BMI $\left(\mathrm{kg} / \mathrm{m}^{2}\right)$ & 0.538 & 1.017 & $0.963 \sim 1.074$ & $0.44 I$ & 1.045 & $0.9934 \sim 1.169$ \\
\hline Hypertension [n (\%)] & 0.913 & 1.036 & $0.55 \mathrm{I} \sim 1.984$ & - & - & - \\
\hline Diabetes [n (\%)] & 0.626 & 1.115 & $0.7|9 \sim 1.73|$ & - & - & - \\
\hline Heart disease [n (\%)] & 0.001 & 2.424 & $1.437 \sim 4.089$ & 0.003 & 2.628 & I.382 4.997 \\
\hline Cerebrovascular disease [n (\%)] & 0.294 & 0.704 & $0.365 \sim 1.357$ & 0.967 & 1.029 & $0.260 \sim 4.071$ \\
\hline $\mathrm{TSH}(\mu \mathrm{lu} / \mathrm{mL})$ & 0.466 & 1.009 & $0.984 \sim 1.035$ & - & - & - \\
\hline TT4 (nmol/L) & $<0.001$ & 0.947 & $0.965 \sim 0.982$ & 0.000 & 0.968 & $0.956 \sim 0.979$ \\
\hline FT4(pmol/L) & 0.011 & 0.911 & $0.848 \sim 0.978$ & 0.056 & 1.282 & $0.993 \sim 1.655$ \\
\hline Hemoglobin $(g / L)$ & 0.038 & 0.990 & $0.980 \sim 0.999$ & 0.102 & 0.922 & $0.838 \sim 1.016$ \\
\hline Serum albumin $(g / L)$ & $<0.001$ & 0.905 & $0.873 \sim 0.939$ & $0.74 I$ & 0.996 & $0.972 \sim 1.021$ \\
\hline Alkaline phosphatase (IU/L) & 0.709 & 1.001 & $0.996 \sim 1.006$ & - & - & - \\
\hline Serum creatinine $(\mu \mathrm{mol} / \mathrm{L})$ & 0.077 & 0.999 & $0.998 \sim 1.000$ & - & - & - \\
\hline Urea nitrogen (mmol/L) & 0.142 & 0.974 & $0.941 \sim 1.009$ & - & - & - \\
\hline Uric acid ( $\mu \mathrm{mol} / \mathrm{L})$ & 0.895 & 1.000 & $0.998 \sim 1.002$ & - & - & - \\
\hline Blood calcium (mmol/L) & $<0.001$ & 0.166 & $0.063 \sim 0.44 \mathrm{I}$ & 0.674 & 0.586 & $0.049 \sim 7.065$ \\
\hline Blood phosphorus (mmol/L) & 0.143 & 0.675 & $0.399 \sim 1.142$ & 0.301 & 0.551 & $0.177 \sim 1.708$ \\
\hline$C$ reactive protein $(\mathrm{mg} / \mathrm{L})$ & 0.236 & 1.006 & $0.996 \sim 1.017$ & - & - & - \\
\hline Serum bilirubin $(\mu \mathrm{mol} / \mathrm{L})$ & 0.002 & 0.864 & $0.789 \sim 0.947$ & 0.339 & 0.874 & $0.664 \sim 1.151$ \\
\hline Aspartate aminotransferase (IU/L) & 0.312 & 0.999 & $0.970 \sim 1.010$ & - & - & - \\
\hline Alanine aminotransferase (IU/L) & 0.019 & 0.979 & $0.962 \sim 0.997$ & 0.165 & 0.952 & $0.889 \sim 1.020$ \\
\hline Parathyroid hormone value $(\mathrm{pg} / \mathrm{mL})$ & 0.852 & 1.000 & $0.999 \sim 1.001$ & - & - & - \\
\hline Low density lipoprotein $C(\mathrm{mmol} / \mathrm{L})$ & 0.537 & 1.073 & $0.858 \sim 1.343$ & - & - & - \\
\hline Triglyceride (mmol/L) & 0.589 & 0.963 & $0.842 \sim 1.013$ & - & - & - \\
\hline Total cholesterol (mmol/L) & 0.389 & 1.077 & $0.909 \sim 1.277$ & - & - & - \\
\hline 24-h urine volume $(\mathrm{mL})$ & 0.022 & 0.999 & $0.999 \sim 1.000$ & 0.606 & 1.000 & $0.999 \sim 1.002$ \\
\hline eGFR (mL/min per $\left.1.73 \mathrm{~m}^{2}\right)$ & 0.259 & 1.027 & $0.981 \sim 1.075$ & 0.811 & 0.986 & $0.880 \sim 1.105$ \\
\hline
\end{tabular}

Notes: FT3 for free triiodothyronine; FT4 for free thyroxine; eGFR for estimated Glomerular Filtration Rate.

the two main thyroid hormones secreted by the thyroid. In the previous studies, T3/T4 ratio seems to be associated with BMI in obese children and adults, also it seems a reliable indicator to predict the effectiveness of treatment and prognosis in lots of diseases. ${ }^{20-22}$ The thyroid secretes only $20 \%$ of $\mathrm{T} 3$, and more than $80 \%$ of $\mathrm{T} 3$ is originated from the deiodination of $\mathrm{T} 4 .{ }^{23}$ The conversion of $\mathrm{T} 4$ to rT3 is an inner-ring deiodination process, which may be stimulated after the occurrence of kidney disease. ${ }^{24}$ Previous study has shown that heart-failure patients with insufficiently treated hypothyroidism have worse renal function than patients with normal or effectively treated hypothyroidism. ${ }^{25}$ Patients with heart disease can stimulate the secretion of catecholamine and others due to stress, leading to a decrease in serum TT3 levels. On the other hand, patients with heart disease often have microinflammatory reactions. Related inflammatory cytokines can act on the hypothalamus-pituitary-thyroid axis through a variety of ways, inhibit the response of pituitary to thyrotropin-releasing hormone and the secretion of 


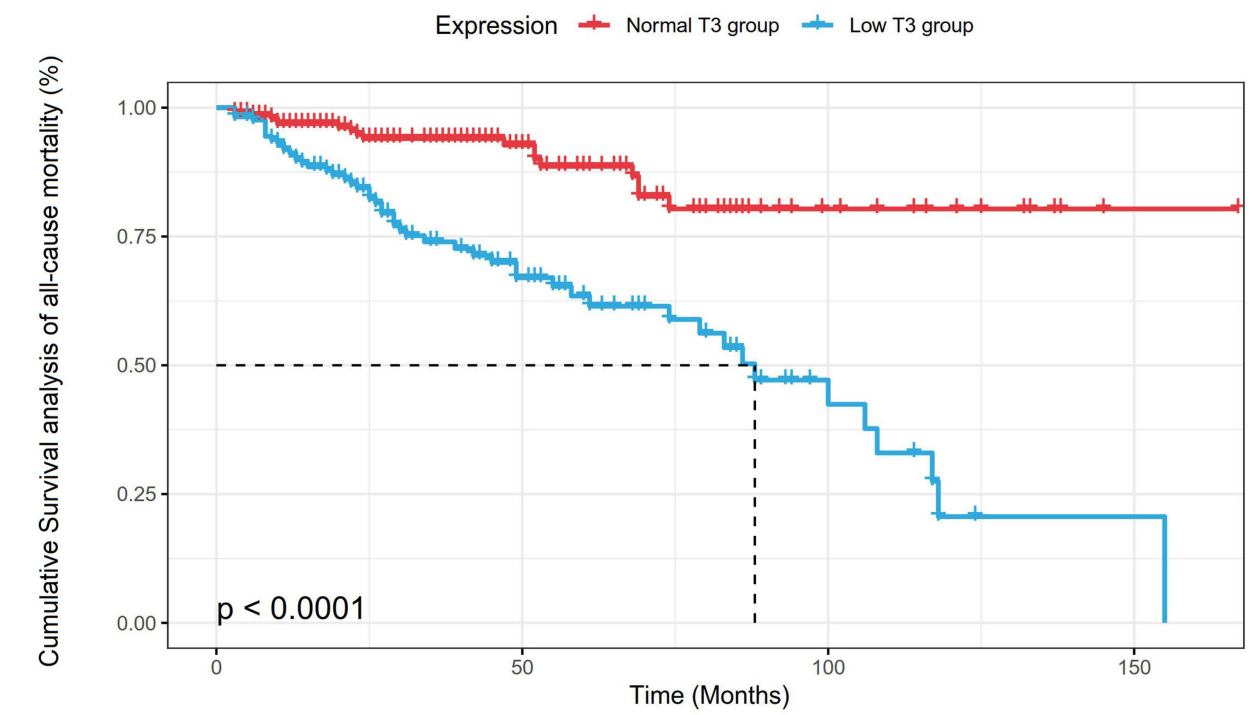

Number at risk (number of events)

\begin{tabular}{r|llll|}
\cline { 3 - 4 } Normal T3 group & $208(0)$ & $72(11)$ & $13(18)$ & $1(18)$ \\
Low T3 group & $168(0)$ & $44(38)$ & $10(47)$ & $1(51)$ \\
\hline
\end{tabular}

Figure I Kaplan-Meier survival analysis of all-cause death in patients with different T3 levels.

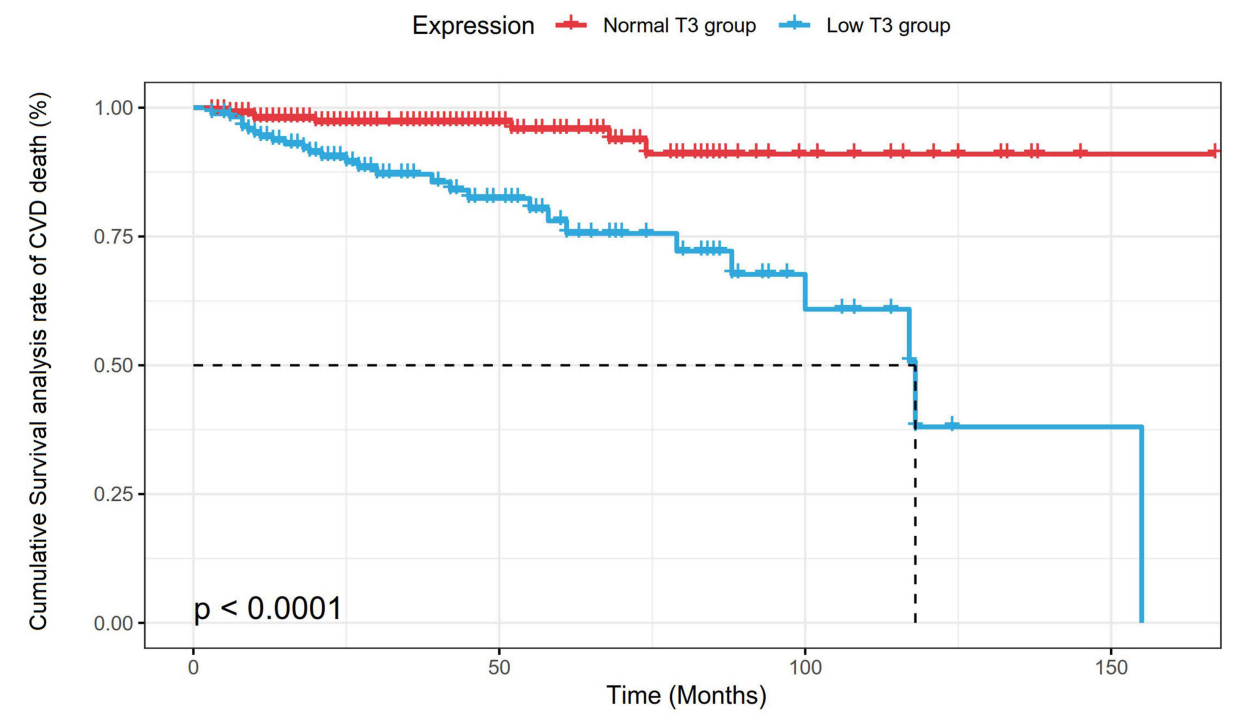

Number at risk (number of events)

\begin{tabular}{c|cccc|}
\cline { 2 - 4 } Normal T3 group & $208(0)$ & $72(5)$ & $13(8)$ & $1(8)$ \\
\hline & $168(0)$ & $44(20)$ & $10(26)$ & $1(28)$ \\
\hline
\end{tabular}

Figure 2 Kaplan-Meier survival analysis of CVD death in patients with different T3 levels. 
Table 3 Cox Regression Analysis Results of the Correlation Between Low Serum T3 Level and All-Cause Death

\begin{tabular}{|c|c|c|c|c|c|c|}
\hline \multirow[t]{2}{*}{ Influencing Factors } & \multicolumn{3}{|c|}{ Univariate Analysis } & \multicolumn{3}{|c|}{ Multivariate Analysis } \\
\hline & $\mathbf{P}$ & HR & $95 \% \mathrm{Cl}$ & $\mathbf{P}$ & HR & $95 \% \mathrm{Cl}$ \\
\hline Gender [male, $\mathrm{n}(\%)]$ & 0.094 & 0.658 & $0.403 \sim 1.074$ & 0.246 & 0.744 & $0.449 \sim 1.231$ \\
\hline Age (years old) & $<0.000$ & 1.072 & $1.05 \mathrm{I} \sim 1.093$ & $<0.001$ & 1.094 & $1.067 \sim 1.121$ \\
\hline Smoking [n (\%)] & 0.173 & 1.465 & $0.846 \sim 2.535$ & - & - & - \\
\hline Drinking [n (\%)] & 0.069 & 1.753 & $0.957 \sim 3.210$ & - & - & - \\
\hline Systolic blood pressure $(\mathrm{mmHg})$ & 0.002 & 1.013 & $1.005 \sim 1.021$ & 0.195 & 1.007 & $0.997 \sim 1.017$ \\
\hline Diastolic blood pressure $(\mathrm{mmHg})$ & 0.285 & 0.992 & $0.977 \sim 1.007$ & - & - & - \\
\hline Heart rate (times) & 0.089 & 1.018 & $0.997 \sim 1.038$ & $<0.001$ & 1.055 & $1.031 \sim 1.080$ \\
\hline Weight (kg) & 0.794 & 0.997 & $0.978 \sim 1.018$ & - & - & - \\
\hline Height (m) & 0.204 & 1.020 & $0.989 \sim 1.053$ & - & - & - \\
\hline BMI $\left(\mathrm{kg} / \mathrm{m}^{2}\right)$ & 0.271 & 0.963 & $0.900 \sim 1.030$ & 0.510 & 0.997 & $0.912 \sim 1.047$ \\
\hline Hypertension [n (\%)] & 0.515 & 0.807 & $0.423 \sim 1.539$ & - & - & - \\
\hline Diabetes [n (\%)] & 0.032 & 1.747 & $1.05 \mathrm{I} \sim 2.905$ & - & - & - \\
\hline Heart disease $[\mathrm{n}(\%)]$ & 0.000 & 3.586 & $2.196 \sim 5.857$ & 0.102 & $\mathrm{I} .584$ & $0.913 \sim 2.748$ \\
\hline Cerebrovascular disease [n (\%)] & 0.029 & 1.571 & $0.777 \sim 3.177$ & 0.608 & 1.206 & $0.590 \sim 2.462$ \\
\hline TSH (mIU/L) & 0.033 & 1.007 & $1.001 \sim 1.013$ & 0.943 & 1.000 & $0.990 \sim 1.009$ \\
\hline TT3 (nmol/L) & 0.001 & 0.303 & $0.154 \sim 0.598$ & 0.053 & 0.475 & $0.224 \sim 1.011$ \\
\hline FT3 (pmol/L) & $<0.001$ & 0.523 & $0.377 \sim 0.724$ & 0.020 & 0.633 & $0.431 \sim 0.930$ \\
\hline TT4 (nmol/L) & 0.057 & 0.992 & $0.984 \sim 1.000$ & - & - & - \\
\hline FT4 (pmol/L) & 0.299 & 0.959 & $0.885 \sim 1.038$ & - & - & - \\
\hline Hemoglobin $(g / L)$ & 0.037 & 0.986 & $0.972 \sim 0.999$ & 0.001 & 0.977 & $0.964 \sim 0.990$ \\
\hline Serum albumin $(g / L)$ & 0.000 & 0.890 & $0.848 \sim 0.935$ & $<0.001$ & 0.894 & $0.845 \sim 0.945$ \\
\hline Alkaline phosphatase (IU/L) & 0.549 & 1.002 & $0.996 \sim 1.008$ & - & - & - \\
\hline Serum creatinine $(\mu \mathrm{mol} / \mathrm{L})$ & 0.000 & 0.997 & $0.996 \sim 0.999$ & 0.599 & 0.999 & $0.997 \sim 1.002$ \\
\hline Urea nitrogen (mmol/L) & 0.736 & 1.008 & $0.960 \sim 1.059$ & - & - & - \\
\hline Uric acid $(\mu \mathrm{mol} / \mathrm{L})$ & 0.110 & 1.002 & $1.000 \sim 1.004$ & - & - & - \\
\hline Blood calcium (mmol/L) & 0.026 & 0.256 & $0.077 \sim 0.853$ & 0.023 & 0.217 & $0.059 \sim 0.807$ \\
\hline Blood phosphorus (mmol/L) & 0.436 & 0.764 & $0.388 \sim 1.505$ & 0.006 & 2.659 & $1.325 \sim 5.337$ \\
\hline$C$ reactive protein $(\mathrm{mg} / \mathrm{L})$ & 0.000 & 1.019 & $1.009 \sim 1.029$ & 0.059 & 1.022 & $0.998 \sim 1.045$ \\
\hline Serum bilirubin $(\mu \mathrm{mol} / \mathrm{L})$ & 0.000 & 0.750 & $0.648 \sim 0.569$ & 0.307 & 0.890 & $0.712 \sim 1.113$ \\
\hline Aspartate aminotransferase (IU/L) & 0.002 & 1.038 & $1.014 \sim 1.064$ & 0.117 & 1.019 & $0.995 \sim 1.043$ \\
\hline Alanine aminotransferase (IU/L) & 0.293 & 0.988 & $0.966 \sim 1.010$ & - & - & - \\
\hline Parathyroid hormone value $(\mathrm{pg} / \mathrm{mL})$ & 0.897 & 1.000 & $0.999 \sim 1.001$ & - & - & - \\
\hline Low density lipoprotein $C(\mathrm{mmol} / \mathrm{L})$ & 0.214 & 0.754 & $0.483 \sim 1.177$ & - & - & - \\
\hline Triglyceride (mmol/L) & 0.624 & 0.953 & $0.786 \sim 1.156$ & - & - & - \\
\hline Total cholesterol (mmol/L) & 0.577 & 1.119 & $0.753 \sim 1.662$ & - & - & - \\
\hline 24-h urine volume $(\mathrm{mL})$ & 0.000 & 0.999 & $0.998 \sim 0.999$ & 0.112 & 1.000 & $0.999 \sim 1.000$ \\
\hline eGFR $\left(\mathrm{mL} / \mathrm{min}\right.$ per $\left.1.73 \mathrm{~m}^{2}\right)$ & 0.000 & 1.071 & $1.039 \sim 1.104$ & $<0.001$ & 1.124 & $1.065 \sim 1.187$ \\
\hline
\end{tabular}

Notes: TSH for Thyroid-stimulating hormone; TT3 for total triiodothyronine; FT3 for free triiodothyronine; TT4 for total thyroxine; FT4 for free thyroxine; eGFR for estimated Glomerular Filtration Rate.

thyroid-stimulating hormone, and enhance the expression of $\mathrm{T} 3$ receptors and their affinity with $\mathrm{T} 3$, leading to a decrease in serum $\mathrm{T} 3$ levels. ${ }^{26}$

Previous studies have investigated the effect of serum $\mathrm{T} 3$ levels on the prognosis of CKD patients with proteinuria and long-term cardiovascular outcomes in incident PD patients. ${ }^{7,27}$ In this study, we further investigated the correlation between serum T3 levels and all-cause and CVD mortality in PD patients. The results showed that whether it was all-cause death or CVD death, the survival rates of low serum T3 level group were generally lower than that of normal serum T3 level group. Low serum FT3 level was an independent risk factor for all-cause death in PD patients $(H R=0.633,95 \% \mathrm{CI}=0.431-0.930 ; P<0.020)$, which was partially consistent with the results of literature studies. ${ }^{14,28}$ Above mentioned results confirmed that low serum T3 level was an independent risk factor for poor prognosis in PD patients. According to the related 
Table 4 Cox Regression Analysis Results of the Correlation Between Low Serum T3 Level and CVD Death

\begin{tabular}{|c|c|c|c|c|c|c|}
\hline \multirow[t]{2}{*}{ Influencing Factors } & \multicolumn{3}{|c|}{ Univariate Analysis } & \multicolumn{3}{|c|}{ Multivariate Analysis } \\
\hline & $\mathbf{P}$ & HR & $95 \% \mathrm{Cl}$ & $\mathbf{P}$ & HR & $95 \% \mathrm{Cl}$ \\
\hline Gender [male, n (\%)] & 0.206 & 0.647 & $0.329 \sim 1.272$ & 0.171 & 0.569 & $0.254 \sim 1.276$ \\
\hline Age (years old) & 0.000 & 1.086 & $1.055 \sim 1.117$ & 0.000 & 1.064 & $1.032 \sim 1.097$ \\
\hline Smoking [n (\%)] & 0.593 & 1.240 & $0.564 \sim 2.724$ & - & - & - \\
\hline Drinking [n (\%)] & 0.497 & 0.663 & $0.203 \sim 2.168$ & - & - & - \\
\hline Systolic blood pressure $(\mathrm{mmHg})$ & 0.028 & 0.975 & $0.953 \sim 0.997$ & 0.663 & 1.000 & $0.998 \sim 1.003$ \\
\hline Diastolic blood pressure $(\mathrm{mmHg})$ & 0.377 & 1.013 & $0.984 \sim 1.043$ & - & - & - \\
\hline Heart rate (times) & 0.006 & 1.015 & $1.004 \sim 1.026$ & 0.003 & 1.044 & $1.015 \sim 1.075$ \\
\hline Weight (kg) & 0.839 & 0.966 & $0.693 \sim 1.347$ & - & - & - \\
\hline Height (m) & 0.796 & 1.032 & $0.812 \sim 1.312$ & - & - & - \\
\hline BMI $\left(\mathrm{kg} / \mathrm{m}^{2}\right)$ & 0.869 & 1.079 & $0.439 \sim 2.649$ & 0.641 & 0.976 & $0.882 \sim 1.082$ \\
\hline Hypertension [n (\%)] & 0.223 & 0.562 & $0.222 \sim 1.422$ & - & - & - \\
\hline Diabetes [n (\%)] & 0.016 & 2.421 & I.I77 4.979 & $0.64 I$ & 0.976 & $0.882 \sim 1.080$ \\
\hline Heart disease [n (\%)] & $<0.001$ & 11.817 & $5.862 \sim 23.821$ & $<0.001$ & 8.970 & $3.824 \sim 21.046$ \\
\hline Cerebrovascular disease [n (\%)] & 0.626 & 1.297 & $0.456 \sim 3.683$ & 0.516 & 0.685 & $0.219 \sim 2.144$ \\
\hline TSH (mIU/L) & 0.817 & 0.988 & $0.891 \sim 1.096$ & - & - & - \\
\hline TT3 (nmol/L) & 0.068 & $0.35 \mathrm{I}$ & $0.114 \sim 1.080$ & - & - & - \\
\hline FT3 (pmol/L) & 0.852 & 1.054 & $0.608 \sim 1.826$ & - & - & - \\
\hline TT4 (nmol/L) & 0.238 & 0.992 & $0.978 \sim 1.006$ & - & - & - \\
\hline FT4 (pmol/L) & 0.656 & 1.028 & $0.909 \sim 1.163$ & - & - & - \\
\hline Hemoglobin $(g / L)$ & 0.093 & 0.984 & $0.966 \sim 1.003$ & - & - & - \\
\hline Serum albumin $(g / L)$ & 0.000 & 0.878 & $0.823 \sim 0.935$ & 0.004 & 0.894 & $0.828 \sim 0.966$ \\
\hline Alkaline phosphatase (IU/L) & 0.894 & 1.001 & $0.991 \sim 1.010$ & - & - & - \\
\hline Serum creatinine $(\mu \mathrm{mol} / \mathrm{L})$ & 0.062 & 1.003 & $1.000 \sim 1.006$ & - & - & - \\
\hline Urea nitrogen $(\mathrm{mmol} / \mathrm{L})$ & 0.345 & 0.969 & $0.908 \sim 1.034$ & - & - & - \\
\hline Uric acid $(\mu \mathrm{mol} / \mathrm{L})$ & 0.001 & 1.055 & $1.023 \sim 1.088$ & 0.006 & 1.005 & $1.002 \sim 1.009$ \\
\hline Blood calcium (mmol/L) & 0.000 & 0.036 & $0.008 \sim 0.155$ & 0.018 & 0.088 & $0.012 \sim 0.663$ \\
\hline Blood phosphorus (mmol/L) & 0.749 & $0.86 \mathrm{I}$ & $0.343 \sim 2.159$ & - & - & - \\
\hline$C$ reactive protein $(\mathrm{mg} / \mathrm{L})$ & 0.035 & 1.015 & $1.001 \sim 1.029$ & 0.086 & 1.025 & $0.997 \sim 1.054$ \\
\hline Serum bilirubin $(\mu \mathrm{mol} / \mathrm{L})$ & 0.001 & 0.716 & $0.582 \sim 0.880$ & 0.704 & 0.960 & $0.775 \sim 1.187$ \\
\hline Aspartate aminotransferase (IU/L) & 0.010 & 1.030 & $\mathrm{I} .007 \sim 1.054$ & 0.707 & 0.920 & $0.596 \sim 1.420$ \\
\hline Alanine aminotransferase (IU/L) & 0.210 & 0.978 & $0.945 \sim 1.013$ & - & - & - \\
\hline Parathyroid hormone value $(\mathrm{pg} / \mathrm{mL})$ & 0.995 & 1.000 & $0.998 \sim 1.002$ & - & - & - \\
\hline Low density lipoprotein $C(\mathrm{mmol} / \mathrm{L})$ & 0.221 & 0.788 & $0.539 \sim 1.154$ & - & - & - \\
\hline Triglyceride (mmol/L) & 0.005 & 1.061 & $1.018 \sim 1.105$ & 0.009 & 1.324 & $1.073 \sim 1.634$ \\
\hline Total cholesterol (mmol/L) & 0.885 & 0.987 & $0.823 \sim 1.182$ & - & - & - \\
\hline $24-h$ urine volume $(\mathrm{mL})$ & 0.006 & 0.999 & $0.840 \sim 1.306$ & - & - & - \\
\hline eGFR $\left(\mathrm{mL} / \mathrm{min}\right.$ per $\left.1.73 \mathrm{~m}^{2}\right)$ & 0.527 & 0.893 & $0.628 \sim 1.269$ & 0.692 & 0.971 & $0.840 \sim 1.123$ \\
\hline
\end{tabular}

Notes: TSH for Thyroid-stimulating hormone; TT3 for total triiodothyronine; FT3 for free triiodothyronine; TT4 for total thyroxine; FT4 for free thyroxine; eGFR for estimated Glomerular Filtration Rate.

mechanism of low serum T3 level and of all-cause death or CVD death in PD patients, low serum FT3 level increases the risk of all-cause death in PD patients, which may be related to the following reasons: (1) low serum FT3 level means that the myocardial contractility of body and the cardiac output are reduced, also leads to an increase in atrial natriuretic peptide content and water and sodium retention, which stimulate the occurrence and aggravation of heart failure, ${ }^{29}$ thereby increasing the risk of death; (2) malnutrition is closely related to the death of PD patients. Patients with low serum FT3 levels usually have varying degrees of malnutrition. In this study, patients with low serum T3 levels had lower hemoglobin and serum albumin, which also indicated that PD patients with malnutrition or low serum T3 levels have a poor prognosis. ${ }^{30}$ The multivariate Cox regression model also showed that serum albumin was an independent risk factor for all-cause death and CVD death in PD patients. Erhan 
et $\mathrm{al}^{31}$ pointed out that the reduction of serum FT3 level in ESRD patients may participate in carotid atherosclerosis through various mechanisms such as endothelial injury, cardiac abnormalities and inflammation. This may be one of the reasons for the increased risk of all-cause death in patients with low serum FT3 levels.

At the same time, the different from previous studies is that although this study shows that low serum T3 levels have a certain relationship with the CVD deaths in PD patients, taking CVD mortality of PD patients, the cumulative CVD mortality in the low serum T3 group was significantly higher than that of patients with normal T3 levels when CVD mortality is taken as the outcome. However, further multivariate Cox regression analysis showed that low serum TT3 and FT3 levels were not risk factors for CVD death in PD patients. The reasons for the differences may be related to the different sources of the study samples, and regional factors such as dietary habits and water quality may affect the thyroid hormone levels in PD patients. ${ }^{32-34}$

It should be pointed out that this study has certain limitations. In this study, the research data comes from a single center. And this is a retrospective study that can only establish the correlation. Moreover, we cannot assess the impact of the dynamic changes of serum T3 level on the prognosis of PD patients In addition, since observational studies cannot assess whether elevated serum T3 levels can improve the prognosis of patients, it still needs to be further confirmed in follow-up studies.

\section{Conclusions}

In summary, this study explored the correlation between baseline serum T3 levels and all-cause deaths and CVD deaths in PD patients, and the results showed that low serum T3 levels in PD patients were related to heart disease and TT4 levels. Low serum FT3 levels were associated with the risk of all-cause death in PD patients, and low serum T3 levels were not significantly associated with CVD death. Serum T3 levels could be helpful for clinicians to predict the prognosis of PD patients. However, it needs to be confirmed by further prospective, observational, multicenter, large-sample study.

\section{Data Sharing Statement}

The datasets used or analyzed during in this study are available from the corresponding author on reasonable request.

\section{Ethics Approval and Informed Consent}

This study was approved by the ethics committee of Hwa Mei Hospital, University of Chinese Academy of Sciences (No.PJ-KY-NBEY-2016-016-01) and complied with the principles of the Declaration of Helsinki for medical research.

\section{Consent for Publication}

Patient consent was waived because of the retrospective nature of the study and the analysis used anonymous clinical data.

\section{Author Contributions}

All authors made substantial contributions to conception and design, acquisition of data, or analysis and interpretation of data; took part in drafting the article or revising it critically for important intellectual content; agreed to submit to the current journal; gave final approval of the version to be published; and agree to be accountable for all aspects of the work.

\section{Funding}

Supported by Ningbo Technology and Public Welfare Foundation, China (Grant No. 2015C50038) and Zhejiang Medical Science and Technology Research Foundation, China, (Grant No. 2020KY258).

\section{Disclosure}

The authors declare that they have no conflicts of interest.

\section{References}

1. Srivastava S, Rajput J, Shrivastava M, Chandra R, Gupta M, Sharma R. Correlation of thyroid hormone profile with biochemical markers of renal function in patients with undialyzed chronic kidney disease. Indian $J$ Endocrinol Metab. 2018;22:316-320. PMID: 30090721. doi:10.4103/ijem.IJEM_475_17

2. Kim SH, Min HK, Lee SW. Relationship between thyroid and kidney function: analysis from the korea national health and nutrition examination survey between 2013 and 2015. Kidney Blood Press Res. 2020;45:442-454. PMID: 32369813. doi:10.1159/000507290

3. Iglesias P, Bajo MA, Selgas R, Díez JJ. Thyroid dysfunction and kidney disease: an update. Rev Endocr Metab Disord. 2017;18:131-144. PMID: 27864708 10.1007/s11154-016-9395-7.

4. Narasaki Y, Sohn P, Rhee CM. The interplay between thyroid dysfunction and kidney disease. Semin Nephrol. 2021;41:133-143. PMID: 34140092. doi:10.1016/j.semnephrol.2021.03.008

5. Schultheiss UT, Daya N, Grams ME, et al. Thyroid function, reduced kidney function and incident chronic kidney disease in a community-based population: the atherosclerosis risk in communities study. Nephrol, Dialysis, Transpl. 2017;32:1874-1881. PMID: 27540046. doi:10.1093/ndt/gfw301 
6. Li J, Wu X, Luo M, et al. Association of serum thyroid hormones with the risk and severity of chronic kidney disease among 3563 chinese adults. Med Sci Monitor. 2020;26:e922910. PMID: 32569261. doi:10.12659/msm.922910

7. Chang TI, Nam JY, Shin SK, Kang EW. Low triiodothyronine syndrome and long-term cardiovascular outcome in incident peritoneal dialysis patients. Clin J Am Soc Nephrol. 2015;10:975-982. PMID: 25979970. doi:10.2215/cjn.03350414

8. Prado-Uribe MDC, Ventura MD, Ávila-díaz M, et al. Low triiodothyronine is associated with elevation of $\mathrm{N}$-terminal pro-brain natriuretic peptide (NT-proBNP) and mortality in dialysis patients. Nefrologia. 2017;37:598-607. PMID: 28755902. doi:10.1016/j. nefro.2017.05.015

9. Dan-yan M, Xiao-rong L, Zhen-yuan L, et al. Low triiodothyronine syndrome can predict poor prognosis in peritoneal dialysis patients. J Shanghai Jiaotong Univ (Med Sci). 2017;37:1500-1505. doi:10.3969/j.issn.1674-8115.2017.11.009

10. Xin-kui T, Ling-ling L, Xin-hong L, Wen T, Qing-feng H. Low triiodothyronine syndrome correlated with residual renal function and survival in patients undergoing maintenance peritoneal dialysis. Chin J Blood Purif. 2020;19:95-98. doi:10.3969/j.issn.16714091.2020.02.007

11. Maraj M, Kuśnierz-Cabala B, Dumnicka. Malnutrition, inflammation, atherosclerosis syndrome (MIA) and diet recommendations among end-stage renal disease patients treated with maintenance hemodialysis. Nutrients. 2018:10. PMID: 29324682. doi:10.3390/ nu10010069

12. von Schacky C. Cardiovascular disease prevention and treatment. Prostaglandins Leukot Essent Fatty Acids. 2009;81:193-198. PMID: 19520557. doi:10.1016/j.plefa.2009.05.009

13. Kuriachan VP, Sumner GL, Mitchell LB. Sudden cardiac death. Curr Probl Cardiol. 2015;40:133-200. PMID: 25813838. doi:10.1016/j. cpcardiol.2015.01.002

14. Sánchez V, Paniagua R, Prado MD, et al. High prevalence of diastolic dysfunction in incident patients on peritoneal dialysis: association with low thyroid hormones. Nephron. 2017;135:120-128. PMID: 27842310. doi: $10.1159 / 000452342$

15. Verger MF, Verger C, Hatt-Magnien D, Perrone F. Relationship between thyroid hormones and nutrition in chronic renal failure. Nephron. 1987;45:211-215. PMID: 3494956. doi:10.1159/ 000184119

16. Musavi Mehdiabadi F, Rashidinejad A, Mahdavi A, Dehesh T. Relationship between thyroid hormones and left ventricular mass in peritoneal dialysis patients. Iran J Kidney Dis. 2020;14:380-388. PMID: 32943593.

17. Meuwese CL, Carrero JJ, Cabezas-Rodríguez I, et al. Nonthyroidal illness: a risk factor for coronary calcification and arterial stiffness in patients undergoing peritoneal dialysis? $J$ Intern Med. 2013;274:584-593. PMID: 23815158. doi:10.1111/joim.12107

18. Kannan L, Shaw PA, Morley MP, et al. Thyroid dysfunction in heart failure and cardiovascular outcomes. Circ Heart Fail. 2018;11: e005266. PMID: 30562095. doi:10.1161/circheartfailure.118.005266

19. Maher SK, Wojnarowicz P, Ichu TA, et al. Rethinking the biological relationships of the thyroid hormones, 1-thyroxine and 3,5,3'triiodothyronine. Comp Biochem Physiol Part D Genomics Proteomics. 2016;18:44-53. PMID: 27085304. doi:10.1016/j. cbd.2016.04.002

20. Bertani L, Tricò D, Pugliese D, et al. Serum triiodothyronine-tothyroxine (T3/T4) ratio predicts therapeutic outcome to biological therapies in elderly IBD patients. Aliment Pharmacol Ther. 2021;53:273-280. PMID: 33210332. doi:10.1111/apt.16167
21. Emokpae MA, Obazelu PA. The association of triiodothyronine-tothyroxine ratio with body mass index in obese nigerian children and adolescents. Med Sci. 2017;5. PMID: 29244733. doi:10.3390/ medsci5040036

22. Michaelsson LF, Medici BB, la Cour JL, et al. Treating hypothyroidism with thyroxine/triiodothyronine combination therapy in denmark: following guidelines or following trends? Eur Thyroid J. 2015;4:174-180. PMID: 26558234. doi:10.1159/000437262

23. Nagao H, Sasaki M, Imazu T, Takahashi K, Aoki H, Minato K. Effects of triiodothyronine on turnover rate and metabolizing enzymes for thyroxine in thyroidectomized rats. Life Sci. 2014;116:74-82. PMID: 25261596. doi:10.1016/j.lfs.2014.09.016

24. Rhee CM. The interaction between thyroid and kidney disease: an overview of the evidence. Curr Opin Endocrinol Diabetes Obes. 2016;23:407-415. PMID: 27428519. doi:10.1097/ med.0000000000000275

25. Merla R, Martinez JD, Martinez MA, et al. Hypothyroidism and renal function in patients with systolic heart failure. Texas Heart Institute J. 2010;37:66-69. PMID: 20200629.

26. Iacoviello M, Parisi G, Gioia MI, et al. Thyroid disorders and prognosis in chronic heart failure: a long-term follow-up study. Endocr Metab Immune Disord Drug Targets. 2020;20:437-445. PMID: 31656160. doi:10.2174/1871530319666191018134524

27. Yang JW, Han ST, Song SH, et al. Serum T3 level can predict cardiovascular events and all-cause mortality rates in CKD patients with proteinuria. Ren Fail. 2012;34:364-372. PMID: 22260378. doi: $10.3109 / 0886022 x .2011 .647342$

28. Sanai T, Nagashima A, Okamura K, et al. Thyroid function in patients on continuous ambulatory peritoneal dialysis in comparison with chronic kidney disease. Clin Nephrol. 2018;89:181-186. PMID: 29035196. doi: $10.5414 / \mathrm{cn} 109228$

29. Peng S, Sun X, Wang X, et al. Myeloid related proteins are up-regulated in autoimmune thyroid diseases and activate toll-like receptor 4 and pro-inflammatory cytokines in vitro. Int Immunopharmacol. 2018;59:217-226. PMID: 29656212. doi:10.1016/j.intimp.2018.04.009

30. Jagadeswaran D, Indhumathi E, Hemamalini AJ, Sivakumar V, Soundararajan P, Jayakumar M. Inflammation and nutritional status assessment by malnutrition inflammation score and its outcome in pre-dialysis chronic kidney disease patients. Clin nutr. 2019;38:341-347. PMID: 29398341. doi:10.1016/j.clnu.2018.01.001

31. Tatar E, Kircelli F, Asci G, et al. Associations of triiodothyronine levels with carotid atherosclerosis and arterial stiffness in hemodialysis patients. Clin j Am Soc Nephrol. 2011;6:2240-2246. PMID: 21836150. doi: $10.2215 / \mathrm{cjn} .02540311$

32. Wang M, Liu L, Li H, et al. Thyroid function, intelligence, and low-moderate fluoride exposure among Chinese school-age children. Environ Int. 2020;134:105229. PMID: 31698198. doi:10.1016/j.envint.2019.105229

33. Cui Y, Yu J, Zhang B, Guo B, Gao T, Liu H. The relationships between thyroid-stimulating hormone and/or dopamine levels in peripheral blood and IQ in children with different urinary iodine concentrations. Neurosci Lett. 2020;729:134981. PMID: 32344109. doi:10.1016/j.neulet.2020.134981

34. Shaik N, Shanbhog R, Nandlal B, Tippeswamy HM. Fluoride and thyroid function in children resident of naturally fluoridated areas consuming different levels of fluoride in drinking water: an observational study. Contemp Clin Dent. 2019;10:24-30. PMID: 32015637. doi:10.4103/ccd.ccd_108_18 


\section{Publish your work in this journal}

Therapeutics and Clinical Risk Management is an international, peerreviewed journal of clinical therapeutics and risk management, focusing on concise rapid reporting of clinical studies in all therapeutic areas, outcomes, safety, and programs for the effective, safe, and sustained use of medicines. This journal is indexed on PubMed Central, CAS,
EMBase, Scopus and the Elsevier Bibliographic databases. The manuscript management system is completely online and includes a very quick and fair peer-review system, which is all easy to use. Visit http://www.dovepress.com/testimonials.php to read real quotes from published authors.

Submit your manuscript here: https://www.dovepress.com/therapeutics-and-clinical-risk-management-journal 